\title{
Inhibitory Effect of Gualou Guizhi Decoction on Microglial Inflammation and Neuron Injury by Promoting Anti-Inflammation via Targeting mmu-miR-155
}

\author{
Haixia Hu $\mathbb{D}^{1,2}$ Xinghua Zhong $\mathbb{D}^{1,2}$ Xinjun Lin $\mathbb{D},{ }^{3}$ Jinbo Yang $\mathbb{D},{ }^{1,2}$ and Xiaoqin Zhu $\mathbb{D}$ 1,2 \\ ${ }^{1}$ Academy of Integrative Medicine, Fujian University of Traditional Chinese Medicine, Fuzhou, China \\ ${ }^{2}$ Fujian Key Laboratory of Integrative Medicine on Geriatrics, Fuzhou, China \\ ${ }^{3}$ Institute of Integrated Chinese and Western Medicine, Fujian University of Traditional Chinese Medicine, Fuzhou, China
}

Correspondence should be addressed to Xinjun Lin; 18960878167@163.com

Received 11 April 2021; Revised 2 July 2021; Accepted 11 August 2021; Published 18 August 2021

Academic Editor: Rusliza Basir

Copyright (c) 2021 Haixia Hu et al. This is an open access article distributed under the Creative Commons Attribution License, which permits unrestricted use, distribution, and reproduction in any medium, provided the original work is properly cited.

Gualou Guizhi decoction (GLGZD) treatment exerts neuroprotective effects and promotes spasticity following ischemic stroke. However, the molecular mechanism of GLGZD treatment on ischemic stroke remains unclear. Our previous study indicated that GLGZD ameliorates neuronal damage caused by secondary inflammatory injury induced by microglia. In the present study, we investigate the potential mechanism of GLGZD treatment on neuron damage induced by neuroinflammation via $\mathrm{mmu}-\mathrm{miR}-155$ in vitro. The HT22 cell line and the BV2 cell line were exposed to oxygen/glucose-deprive (OGD) conditions; the conditioned medium was prepared using the supernatants from OGD-stimulated BV2 cells after pretreating with GLGZD. Cell viability was determined by MTT assays; levels of released inflammatory cytokines were assessed using the BioPlex system. mmu-miR-155 and its targeting genes were detected using real-time reverse transcription polymerase chain reaction (RT-PCR). The expression of anti-inflammatory proteins was evaluated by Western blotting. DAPI staining was used to test the apoptotic cells. Our results showed that GLGZD pretreatment significantly induced IL10 release and decreased the production of TNF- $\alpha$, IL6, and IFN- $\gamma$. In addition, GLGZD markedly attenuated mmu-miR-155 expression and its downstream SOCS1, SMAD2, SHIP1, and TAB2 expression levels. The DAPI-stained apoptotic cell death and caspase- 3 activation in HT22 cells exposed to the conditioned medium were reversed by GLGZD treatment. Our findings suggested that GLGZD pretreatment downregulates the mmu-miR155 signaling, which inhibits microglial inflammation, thereby resulting in the suppression of neuron apoptosis after OGD stress. The underlying mechanisms may provide the support for GLGZD treatment of cerebral ischemic injury.

\section{Introduction}

Ischemic stroke remains a leading cause that challenge public health worldwide. Microglia are the major immune cells in the nervous system, which can be activated and involved in the neuropathological development $[1,2]$. The molecules released from injured brain tissue provoke microglial activation and generate a ring of cytokines, some of which exacerbate primary brain damage, and others exert anti-inflammatory effects [3-5]. mmu-miR-155, a highly expressed miRNA in the CNS, is reported to be a neuroinflammatory regulator targeting different mediators, SHIP1, SOCS1, SMAD2, and TAB2, which involve in inflammatory balance [6, 7]. Mmu-miR-155 promotes inflammatory reaction by suppressing SHIP1, SOCS1, SMAD2, and TAB2 expressions, all of which inhibit inflammation by decreased expressions of proinflammatory cytokines and increased expressions of anti-inflammatory cytokines $[8,9]$. Given the significance of mmu-miR-155 in the regulation of neuroinflammation, the treatment targeting mmu-miR-155 in ischemic cerebral injury will be identified as a promising therapy for ischemic stroke.

Gualou Guizhi decoction (GLGZD) is a commonly used traditional Chinese medicine and prescribed in the "Essentials from the Golden Cabinet," which is a traditional Chinese medical work written by Zhongjing Zhang in Han Dynasty. It has been used for clinical treatment of spasticity following ischemic stroke and mainly used at The Fujian 
University of the TCM Affiliated Second People's Hospital (Fuzhou, China). GLGZD's recipe includes Radix Trichosanthis, Ramulus Cinnamomi, Radix Paeoniae Alba, Radix Glycyrrhizae, Rhizoma Zingiberis Recens, and Fructus Ziziphi Jujubae. The neuroprotective effect of GLGZD may involve a number of different mechanisms, including reduced inflammatory cytokine levels, an antioxidation effect, and the modulation of Glu levels. Based on our previous study, GLGZD has been shown to possess multiple pharmacological properties to reduce inflammation and protect neuron from severe injury. However, the underlying mechanisms of treatment remain unclear. The present study attempted to explore the anti-inflammatory effects of GLGZD on OGD-induced BV2 activation regulated by mmu-miR-155 signaling and the neuroprotective role of GLGZD in HT22 cell apoptosis stimulated by microglial conditioned medium; this study will further reveal the underlying possible mechanisms of GLGZD treatment for cerebral ischemia injury.

\section{Materials and Methods}

2.1. GLGZD Preparation. The prescribed herbal medicine were obtained from Guoyitang Drug Store (Fuzhou, China). The decoction ingredients and GLGZD extracts preparation were applied in this experiment according to our previous study $[10,11]$. The working concentrations for treatment of GLGZD were $100-400 \mu \mathrm{g} / \mathrm{ml}$.

2.2. HT22 and BV2 Cell Culture. Immortalized mouse hippocampal neuron HT22 cells and the murine BV2 microglial cell line were grown in high-glucose Dulbecco's modified Eagle's medium (DMEM, Gibco, Carlsbad, USA) supplemented with $10 \%$ fetal bovine serum (FBS, Gibco) and $1 \%$ penicillin/streptomycin. The cells were maintained at $37^{\circ} \mathrm{C}$ in a humidified atmosphere of $5 \% \mathrm{CO}_{2}$. BV2 cells were pretreated by GLGZD $(100 \mu \mathrm{mol} / \mathrm{L})$ for $2 \mathrm{~h}$ and then stimulated with different treatments.

2.3. Oxygen-Glucose Deprivation Model. BV2 cells were cultured in media deprived from oxygen and glucose to induce OGD condition and then placed in an incubator (H45, Whitely, England) with humidified atmosphere containing $1 \%$ oxygen, $94 \%$ nitrogen, and $5 \% \mathrm{CO}_{2}$ at $37^{\circ} \mathrm{C}$. All cells were subjected to $4 \mathrm{~h}$ OGD followed by incubation of the 24-hour reperfusion period in the normal culture medium. Controls were incubated with the normal culture condition and the identical time as the OGD culture.

2.4. GLGZD Treatment and Preparation of Microglial Conditioned Medium (MCM). GLGZD was extracted and diluted with the culture medium to various concentrations: 100,200 , and $400 \mu \mathrm{g} / \mathrm{ml}$. The BV2 cells were pretreated with different concentration of GLGZD for $2 \mathrm{~h}$ and subsequently exposed to OGD injury. The MCM was derived from the supernatant of BV2 cells subjected to OGD stress and pretreated with or without GLGZD. The MCM was collected and added to HT22 cultures.

2.5. 3-[4, 5-Dimethyl-thiazol-2-yl]-2,5-diphenyltetrazolium Bromide (MTT) Assay. HT22 cells were seeded at the density of $3 \times 10^{4}$ cells/well in a 96-well plate and then cultured overnight, and then, the medium was replaced with the MCM for additional $24 \mathrm{~h}$ incubation. BV2 cells were plated onto 96-well plates $\left(5 \times 10^{4}\right.$ cells/well $)$ and pretreated with the indicated concentrations of GLGZD for $2 \mathrm{~h}$ before OGD stress. The cell viability of BV2 cell subjected to OGD and HT22 exposed to MCM were measured by MTT assay. Briefly, $100 \mu \mathrm{L}$ of $0.05 \%$ MTT reagent (Sigma, St. Louis, MO) was added to each well and incubated at $37^{\circ} \mathrm{C}$ for $4 \mathrm{~h}$ followed by $100 \mu \mathrm{L}$ of DMSO solution to dissolve the formazan crystals. The absorbance at $570 \mathrm{~nm}$ was evaluated using a microplate reader (ElX-880, BioTek Inc., Winooski, VT). The data were expressed as percentages of the control.

2.6. Detection of Cytokines by the BioPlex System. After OGD injury and GLGZD treatment, the BV2 cell culture supernatant was harvested to measure the cytokine concentrations. BioPlex inflammation-related ELISA kits (\#M50OKCAF0Y, BioRad Laboratories, Hercules, CA, USA) were used to measure the secretion levels of a ring of cytokines according to the manufacturer's protocol, including TNF $\alpha$, IL6, IFN- $\gamma$, and IL10. Briefly, add $50 \mu$ l diluted beads to each well of the assay plate, and then, wash the plate with BioPlex wash buffer. After that, add $50 \mu$ l standards, samples, and controls to incubate on the shaker at $850 \mathrm{rpm}$ for $30 \mathrm{~min}$ at RT. The detection antibodies were added to each well followed by washing the plate three times. The plate was incubated at $850 \mathrm{rpm}$ for $30 \mathrm{~min}$ at RT. Then, the streptavidinphycoerythrin was added to each well and incubated at $850 \mathrm{rpm}$ for $10 \mathrm{~min}$ at RT. After resuspending the beads in assay buffer and shaking at $850 \mathrm{rpm}$ for $30 \mathrm{sec}$, the results were determined using BioPlex 200 system (BioRad Laboratories, Hercules, CA, USA) at $450 \mathrm{~nm}$.

2.7. Real-Time PCR. Total RNA was isolated using TRIzol R reagent (Invitrogen, Carlsbad, CA, United States). Reverse transcription to synthesize cDNA was performed using the RevertAid First Strand cDNA Synthesis Kit (Fermentis Life Science, Ontario, Canada). cDNA template was used for the quantitative real-time PCR amplification. Specific primers were designed, and the primer sequences for each gene are as follows: mmu-miR-155-5p: Fwd 5'-CAGTTTGTGGAACGGTGCTG-3'; Rev 5'-TGGTAGGGTCATCGGGT TCT-3'; SOCS1: Fwd 5'-CTGCGGCTTCTATTGGG GAC3', Rev 5'-AAAAGGCAGTCGAAGGTCTCG-3'; SMAD2: Fwd $5^{\prime}$-CATCAGCCAATGGCAAGTGAA-3', Rev 5'-AGA ACAGGGTCTGCATCCATCATA- $3^{\prime}$; SHIP1: Fwd $5^{\prime}$ CAGGGATGAAGTACAACTTGCC-3'; Rev $5^{\prime}$-TCTC CTTCCTGACTCTTGACA-3'; TAB2: Fwd 5'-CGATCAG CTGTTGCGAGCGCTGCAC-3'; Rev $5^{\prime}$-GTGCAGCGCTC GCAACAGCTGATCG-3'; and GAPDH: Fwd 5'-CACA TTGGGGGTAGGAACAC- $3^{\prime}$; $\quad$ Rev $\quad 5^{\prime}$-ACCCAGAAGA 
CTGTGGATGG-3'. RT-PCR was conducted using the following condition: $94^{\circ} \mathrm{C}$ for $30 \mathrm{~s}, 55^{\circ} \mathrm{C}$ for $45 \mathrm{~s}$, and $72^{\circ} \mathrm{C}$ for $1 \mathrm{~min}$ for a total of $35 \mathrm{cycles}, 72^{\circ} \mathrm{C}$ for $10 \mathrm{~min}$. GAPDH was served as an internal control for normalization. The relative expression level of the PCR products was analyzed using the $2^{(-\Delta \Delta \mathrm{Ct})}$ method.

2.8. Western Blot Analysis. After exposure to different treatments, cells were collected and lysed in RIPA-containing protease and phosphatase inhibitors (Roche, Penzberg, Germany) followed by centrifugation at $12,000 \mathrm{~g}$ for $5 \mathrm{~min}$ at $4^{\circ} \mathrm{C}$. The supernatant was collected, and the protein concentration was assessed using the BCA assay (Beyotime, Biotechnology, Beijing, China). $50 \mu \mathrm{g}$ of proteins was separated by SDS-PAGE electrophoresis and transferred to polyvinylidene difluoride (PVDF) membranes (BioRad, Munich, Germany). The membranes were then blocked in $5 \%$ nonfat milk for $1 \mathrm{~h}$ at room temperature to prevent nonspecific binding and incubated overnight at $4^{\circ} \mathrm{C}$ with primary antibodies against SOCS1, SMAD2, SHIP1, and TAB2 (1:1000, Cat. No. 68631 for SOCS1, Cat. No. 5339 for SMAD2, Cat. No. 2725 for SHIP1, and Cat. No. 3745 for TAB2, Cell Signaling Technology, Beverly, MA). Afterward, the membranes were washed with TBS/T (TBS with $0.05 \%$ Tween 20) and incubated with the secondary antibody IgG (1:5000, Cat. No. 91196, HRP Conjugate, Cell Signaling Technology, Beverly, MA) at room temperature for $1 \mathrm{~h}$. The immune-reactive proteins were visualized using the enhanced chemiluminescence (ECL) reagent (P0018, Beyotime Biotechnology). $\beta$-Actin (anti- $\beta$-actin antibody, cat. no. sc47778, Santa Cruz Biotechnology, Inc. Dallas, TX, USA), was used as an internal reference. The data were normalized against the internals reference and then expressed as fold changes compared to the controls, quantified by using BioRad ChemiDoc ${ }^{\mathrm{TM}}$ XRS Image Lab Software.

2.9. DAPIStaining for Nuclei. HT22 cells were cultured on 6well culture plates at a density of $3 \times 10^{5}$ cells $/ \mathrm{mL}$ and treated with MCM as described above. After fixation with $4 \%$ paraformaldehyde at room temperature for $20 \mathrm{~min}$, the cells were stained with $10 \mathrm{mg} / \mathrm{mL}$ 6-diamidino-2-phenylindole dihydrochloride (DAPI; Beyotime Biotechnology, Shanghai, China) for $15 \mathrm{~min}$. The formation of fragmented or condensed DNA was determined as typical characteristic of apoptotic cells. Representative photographs were taken by a fluorescence microscope (Leica Microsystems, Wetzlar, Germany) at $100 \times$ and $200 \times$ magnification. The cell fluorescence was measured using image $J$ software. The percentage of apoptotic cells in total cells was calculated.

2.10. Detection of Caspase-3 Activity. The caspase-3 activity test was carried out according to the instructions of caspase3 activity assay kit (lot no. C1116, Beyotime Biotechnology, Shanghai, China). In brief, the cells were centrifugated and collected for protein extraction and quantification. After that, the Ac-DEVD-pNA (acetyl-Asp-Glu- Val-Asp p-nitroanilide) was added to extract protein to incubate for
90 min till production of yellow PNA (p-nitroaniline). Finally, the absorbance at $405 \mathrm{~nm}$ was detected by a microplate reader (BioTek 8008, Bad Friedrichshall, Germany), and the caspase-3 activity was determined according to the standard curve.

2.11. Statistical Analysis. Three independent experiments were conducted; all results were expressed as mean \pm SD. Statistical analysis was determined by one-way analysis of variance (ANOVA) using SPSS 20.0 software. Differences were considered statistically significant at $p<0.05$.

\section{Results}

3.1. Protective Effects of GLGZD Pretreatment on OGD-Induced Injury in BV2 Cells. As shown in Figure 1, the viability of BV2 cells exposed to OGD insult was measured using the MTT assay, which indicated that exposure to OGD for $4 \mathrm{~h}$ followed by additional $24 \mathrm{~h}$ of reperfusion that resulted in significant decrease of cell viability. However, when compared with the OGD group, pretreatment with GLGZD could significantly inhibit the OGD induced injury in BV2 cells in a concentration dependent manner $(p<0.05)$. Furthermore, the BV2 survival was not obviously affected by GLGZD alone at the concentration from 100 to $400 \mu \mathrm{g} / \mathrm{mL}$.

3.2. GLGZD Pretreatment Regulated OGD-Induced Cytokines Production in BV2 Cells. To investigate the anti-inflammatory function of GLGZD, we performed multiELISA to assess the release of inflammatory cytokines. In our study (Figure 2), compared to the control group, the production of TNF- $\alpha$, IL6, and IFN- $\gamma$ in the culture medium supernatants was significantly enhanced by OGD stress $(p<0.05)$ while reduced by GLGZD pretreatment $(p<0.05$ and 0.01$)$. In contrast, low levels of IL10 productions in the OGD group were increased significantly in the GLGZD $(200 \mu \mathrm{g} / \mathrm{mL})$ group $(p<0.01)$.

3.3. Effect of GLGZD on Expression Levels of $m m u-m i R-155$ and Its Targeting Genes in BV2 Cells under OGD Condition. mmu-miR-155 is a key microRNA that involved cell inflammation poststroke, and previous results indicated that its levels are obviously raised in activated microglia. The present study showed that mmu-miR-155 and its downstream genes were obviously influenced by GLGZD pretreatment in BV2 subjected by OGD. As shown in Figure 3(a), compared with the control group, there was an obvious increase in mmu-miR-155 $(p<0.01)$ and decrease in SOCS1, SMAD2, SHIP1, and TAB2 in the OGD group $(p<0.05$ and $p<0.01)$. However, GLGZD treatment significantly suppressed the upregulation of mmu-miR-155 $(P<0.05)$ and increased SOCS1, SMAD1, SHIP1, and TAB2 mRNA expression induced by OGD $(p<0.05$ and $p<0.01)$. Simultaneously, the protein alteration in different groups were consistent with the mRNA expression results $(p<0.05$ and $p<0.01$, Figure $3(\mathrm{~b})$ ). 


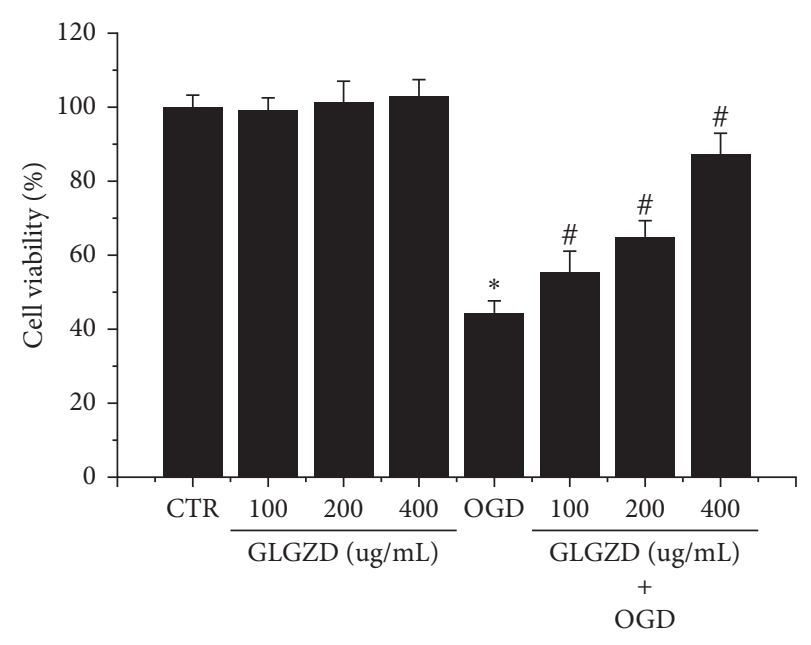

FIGURE 1: effect of GLGZD on the viability of BV2 cells subjected to OGD stimulation. The MTT assay was used to determine the viability of BV2 cells. All data were expressed as mean \pm SD of three independent experiments performed in triplicate. ${ }^{*} P<0.05$ vs. control (CTR) and ${ }^{\#} P<0.05$ vs. OGD condition.

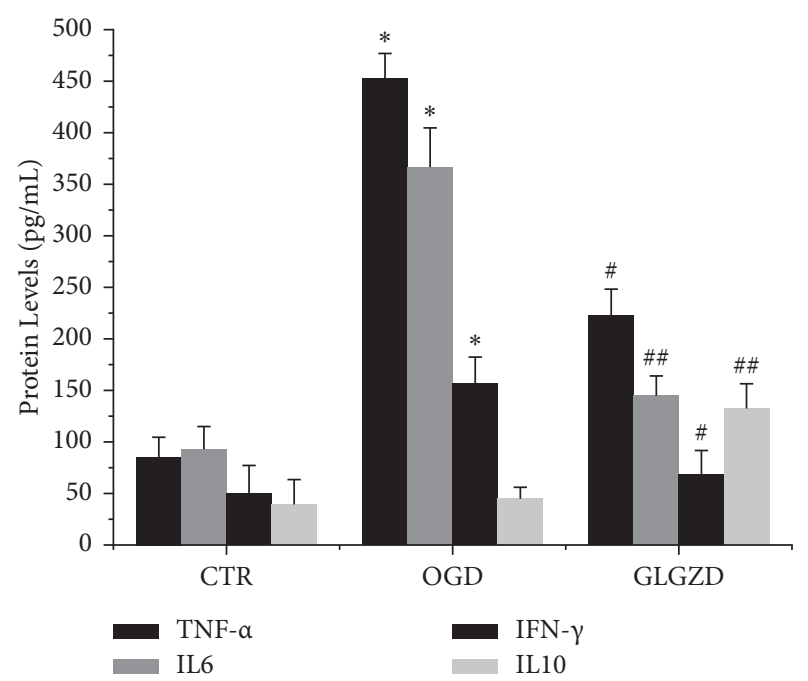

FIGURE 2: The impact of GLGZD on the expression levels of pro and anti-inflammatory cytokines in OGD-stimulated BV2 cells. The protein levels of pro and anti-inflammatory cytokines were analyzed by BioPlex multiple ELISA. Data are expressed as mean \pm SD from three independent experiments. ${ }^{*} P<0.05$ vs. control and ${ }^{\#} P<0.05,{ }^{\#} P<0.01$ vs. OGD condition.

3.4. GLGZD Protected HT22 Cells from the Neurotoxic Microglial Conditioned Medium Induced by OGD. Microglial conditioned medium (MCM) was established and added to HT22 for incubation. Afterward, to examine the neuroprotective potential of GLGZD, the HT22 cell viability was tested using MTT assay, and the cell apoptosis was assessed by DAPI staining. As shown in Figure 4(a), the conditioned medium from activated microglia induced decrease in HT22 cell viability compared with the control group; however, treatment with GLGZD at $200 \mu \mathrm{g} / \mathrm{mL}$ concentration restored the cell survival partially $(p<0.05$, Figure 4(a)). Accordingly, DAPI staining was utilized for detecting HT22 cells of nuclear morphometry; the presence of chromatin condensation and nuclear shrinkage is indicative of the occurrence of apoptosis. Our results showed that there were more apoptotic cells in the MCM-treated group, and GLGZD pretreatment reduced the number of apoptotic cells significantly $(p<0.05$, Figure $4(b))$.

3.5. GLGZD Ameliorated Caspase-3 Activity in MCM-Induced HT22 Cells. Caspase-3, which belongs to caspase family, plays a key role in the process of apoptosis, including chromatin condensation and DNA fragmentation [12]. A significant increase in caspase-3 activity was observed following MCM stimulation in HT22 cells compared with that of the control group $(p<0.05)$. In contrast, pretreatment with GLGZD reversed the influence of OGD injury and significantly reduced caspase- 3 activity in HT22 cells exposed to MCM (Figure 5, $p<0.05$ ).

\section{Discussion}

Ischemic stroke is a neurological disorder resulted from loss of blood supply leading to neuronal damage. Dead neurons generate danger-associated molecular patterns (DAMPs) triggering excessive inflammatory responses $[13,14]$. Microglia are the resident immune cells in the brain and play a central role in the postischemic inflammation. Activated microglia release a wide range of inflammatory mediators including proinflammatory cytokines exacerbating brain injury. On the other hand, anti-inflammatory cytokines are produced by microglia conversely promoting brain repair $[15,16]$. Develop novel immunomodulatory approaches to improve mortality and functional outcome of those inflicted with ischemic stroke. Thus, developments in identifying immunomodulatory approaches will contribute to limit neuronal injury and improve poststroke functional impairment.

Ample evidence indicate that miRNAs potentially involve in many biological processes at the posttranscriptional level, including cell apoptosis and inflammation [17, 18]. Among various miRNAs, miR-155 is considered as the key regulator in postischemic brain inflammation [19]. In addition, miR155 can exert both pro and anti-inflammatory roles by targeting different mediators, such as SHIP1, SOCS1, SMAD2, and TAB2. When ischemia occurs, the expression of mmumiR-155 was significantly upregulated, and inhibiting mmumiR-155 could reduce the secretion of TNF- $\alpha$, IL6, IL $1 \beta$, and IFN- $\gamma$ induced by neuroinflammation $[20,21]$.

Fewer side effects and multitarget therapy are the advantages of traditional Chinese medicine (TCM) in the clinical treatment of stroke. GLGZD is a widely used and classical TCM for approximately more than 10 years as an effective treatment for spasticity poststroke. The molecule mechanism may correlate with the neuron protection from serious damage through antioxidation and antiinflammation [22, 23]. Moreover, GLGZD promotes neurological functional recovery by increasing neuron proliferation and enhancing axonal regeneration following focal cerebral ischemia. In our previous study, GLGZD exhibited significant anti-inflammatory effects on LPS- 

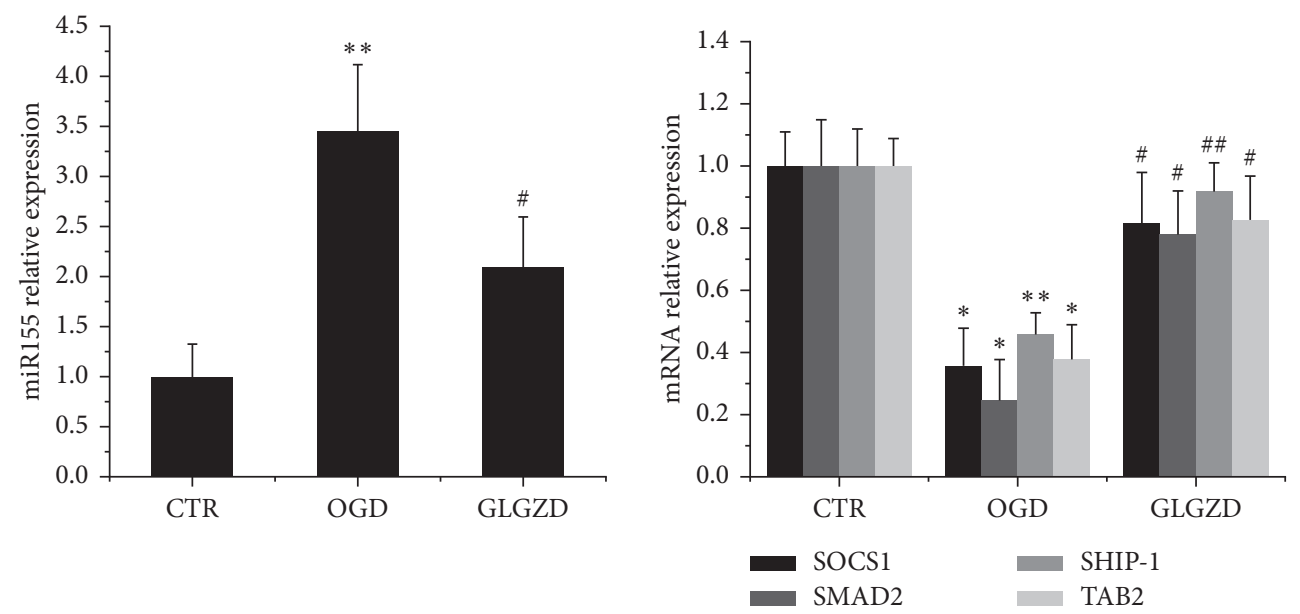

(a)
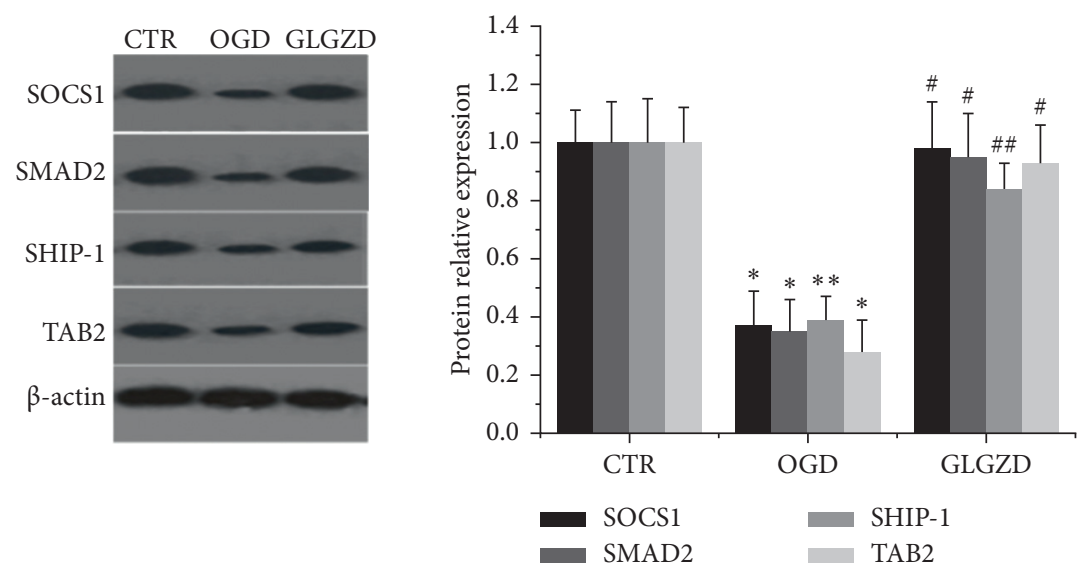

(b)

FiguRE 3: GLGZD prominently restricts the level of mmu-miR-155 and promotes the expression of its targeting meditators. (a) The mRNA levels of mmu-miR-155 and SOCS1, SMAD2, SHIP1, and TAB2 measured by RT-PCR and quantitative assessment performed by normalizing to the level of internal control GAPDH. (b) The protein expression level of SOCS1, SMAD2, SHIP1, and TAB2 assessed by Western blotting assay and normalized to the level of internal control $\beta$-actin. $\beta$-Actin was used for normalization, and the intensity of bands was quantified by densitometric analysis. All values represent mean $\pm \mathrm{SD}$ of three independent experiments. ${ }^{*} P<0.05$, ${ }^{* *} P<0.01$ vs. control and ${ }^{\#} P<0.05,{ }^{\# \#} P<0.01$ vs. OGD condition.

stimulated BV2 cells. Here, we further explore the underlying mechanism of neuroprotective potential of GLGZD pretreatment in the cultured BV2 cells and HT22 cells exposed to OGD injury.

In our present study, we established an OGD model to investigate the function of GLGZD in BV2 and HT22 cells. The results showed that the addition of GLGZD significantly reversed the decreased BV2 cell viability induced by OGD. Furthermore, GLGZD pretreatment blocked the upregulated expression levels of TNF- $\alpha$, IL6, and IFN- $\gamma$ and promoted the expression levels of IL10 induced by OGD insult in BV2 cells. To test whether GLGZD treatment is involved in mediating mmu-miR-155 signaling in inflammatory reaction, mRNA and protein levels of the specific targeting mediators of mmu-miR-155 were detected by RT-PCR and Western blotting assays. We observed a dramatic upregulation in mmu-miR-155 expression in vitro model by exposing BV2 cells to OGD conditions. Simultaneously, the expression levels of its target mediators were affected obviously by GLGZD. Among these mediators, SOCS1, SMAD2, SHIP, and TAB2 enhance the production of antiinflammatory cytokines and reduce the generation of proinflammatory cytokines; however, GLGZD treatment can promote their effects on inflammation significantly. These observations demonstrated that GLGZD not only play a protective role in microglia but also inhibit microglial overactivation. Moreover, the impact of GLGZD was dependent on mmu-miR-155-induced signal in microglia. Then, to further examine the effect of GLGZD on the MCMtreated HT22 cells, the results suggested that the neuron apoptotic cells and caspase-3 activity evoked by OGD insult were decreased by GLGZD treatment. Taken together, our findings clearly revealed that the neuroprotective properties of GLGZD were involved in negative regulation of inflammation via mmu-miR-155 and antiapoptotic role after the induction of OGD.

To sum up, our data confirmed the protective effect of GLGZD on neuron injury induced by inflammatory and 

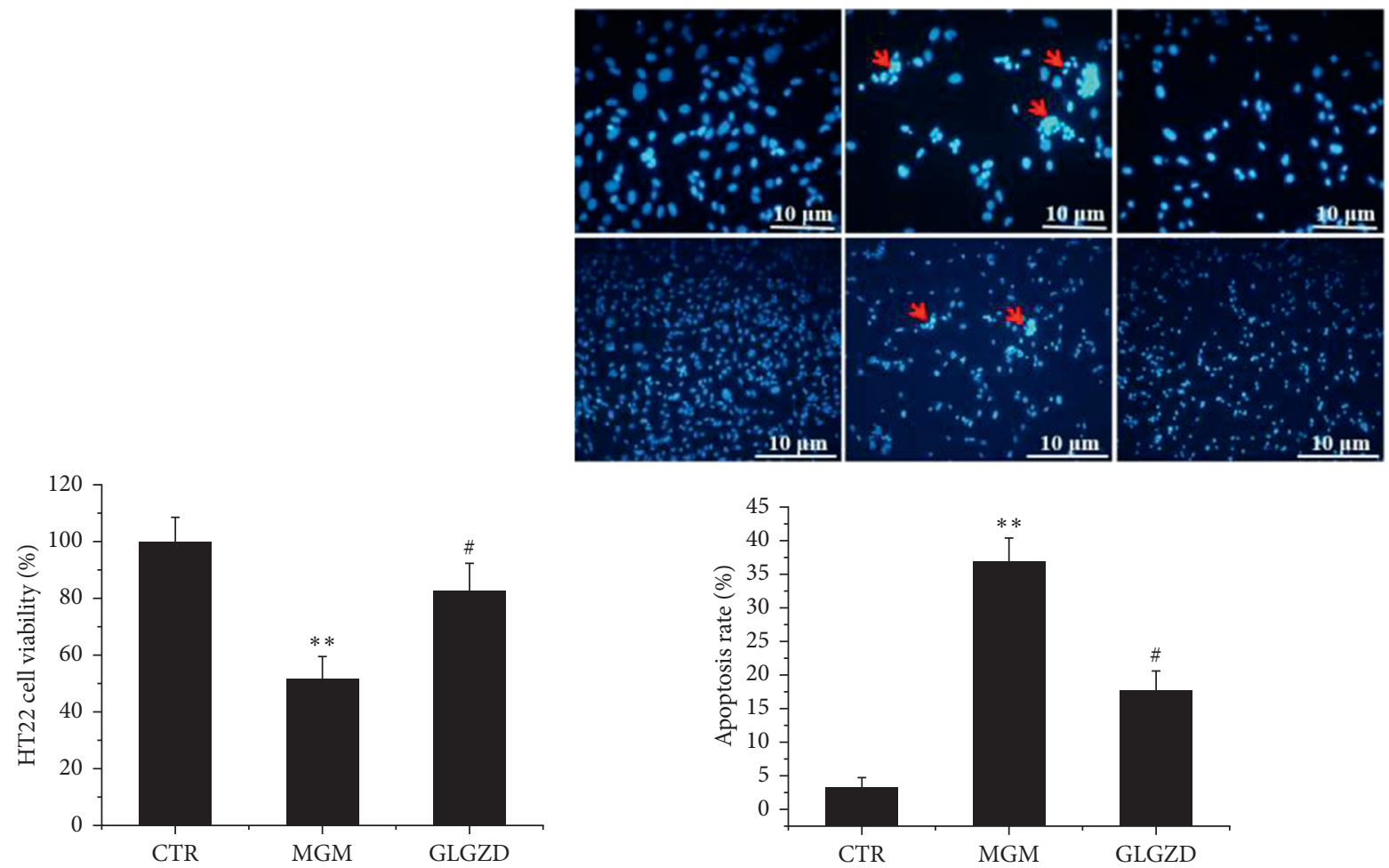

(a)

(b)

FIgURE 4: The neuroprotection of GLGZD on the MCM-induced cytotoxicity in HT22 cells. (a) The cell viability of HT22 cells tested by MTT assay. (b) The apoptotic HT22 cells presented DAPI staining. Arrowheads indicted apoptosis cells and the apoptosis rate was calculated. All data are expressed as mean \pm SD of three independent experiments. ${ }^{* *} P<0.01$ vs. control and ${ }^{\#} P<0.05$ vs. OGD condition.

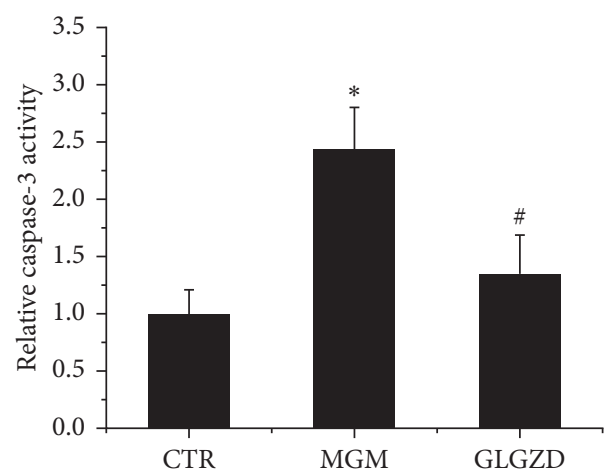

FIGURE 5: GLGZD reverses the activity of caspase-3 in MCM-induced HT22 cells. The caspase-3 activity was assayed by a microplate reader at absorbance of $405 \mathrm{~nm}$. All results were presented as mean \pm SD of three independent experiments. Results represent 3 independent experiments. ${ }^{*} P<0.05$ vs. control and ${ }^{\#} P<0.05$ vs. OGD condition.

apoptotic effects mediated by mmu-miR-155 signaling, thereby providing experimental evidence of GLGZD to alleviate brain damage and improve functional recovery for ischemic stroke therapy.

\section{Abbreviations}

GLGZD: Gualou Guizhi decoction

IL1 $\beta$ : Interleukin- $1 \beta$

IL6: Interleukin-6

IL10: Interleukin-10
OGD: Oxygen-glucose deprivation

TCM: Traditional Chinese medicine

IFN- $\gamma$ : Interferon-gamma

TNF- $\alpha$ : Tumor necrosis factor- $\alpha$

DAPI: $\quad 4^{\prime}, 6$-Diamidino-2-phenylindole

SOCS1: Suppressors of cytokine signaling 1

SMAD2: Signal transduction molecule 2

SHIP1: Src homology 2 (SH2) domain containing inositol polyphosphate 5-phosphatase 1

TAB2: TGF-beta activated kinase 1 (TAK1)/MAP3K7 binding protein 2 . 


\section{Data Availability}

The datasets analyzed to support the findings of this study are available from the corresponding author upon request.

\section{Conflicts of Interest}

The authors declare that there are no conflicts of interest.

\section{Acknowledgments}

This work was funded by open research platform of Fujian University of Traditional Chinese Medicine (Laboratory of Traditional Chinese Medicine Pharmacognosy (Level 3), X2019014-platform) and Fujian Province Natural Science Foundation (2017J01849).

\section{References}

[1] S. Xu, J. Lu, A. Shao, J. H. Zhang, and J. Zhang, "Glial cells: role of the immune response in ischemic stroke," Frontiers in Immunology, vol. 11, p. 294, 2020.

[2] R. Patnala, T. V. Arumugam, N. Gupta, and S. T. Dheen, "HDAC inhibitor sodium butyrate-mediated epigenetic regulation enhances neuroprotective function of microglia during ischemic stroke," Molecular Neurobiology, vol. 54, no. 8, pp. 6391-6411, 2017.

[3] M. Kanazawa, I. Ninomiya, M. Hatakeyama, T. Takahashi, and T. Shimohata, "Microglia and monocytes/macrophages polarization reveal novel therapeutic mechanism against stroke," International Journal of Molecular Sciences, vol. 18, no. 10, p. 2135, 2017.

[4] Y. Ma, J. Wang, Y. Wang, and G.-Y. Yang, "The biphasic function of microglia in ischemic stroke," Progress in Neurobiology, vol. 157, pp. 247-272, 2017.

[5] Z. J. Liu, Y. Y. Ran, S. Y. Qie et al., "Melatonin protects against ischemic stroke by modulating microglia/macrophage polarization toward anti-inflammatory phenotype through STAT3 pathway," CNS Neuroscience and Therapeutics, vol. 25, no. 12, pp. 1353-1362, 2019.

[6] Y. Guo, W. Hong, X. Wang et al., "MicroRNAs in microglia: how do MicroRNAs affect activation, inflammation, polarization of microglia and mediate the interaction between microglia and glioma?" Frontiers in Molecular Neuroscience, vol. 12, p. 125, 2019.

[7] X. Zheng, H. Huang, J. Liu, M. Li, M. Liu, and T. Luo, "Propofol attenuates inflammatory response in LPS-activated microglia by regulating the miR-155/SOCS1 pathway," Inflammation, vol. 41, no. 1, pp. 11-19, 2018.

[8] K. Jiang, J. Yang, S. Guo, G. Zhao, H. Wu, and G. Deng, "Peripheral circulating exosome-mediated delivery of miR155 as a novel mechanism for acute lung inflammation," Molecular Therapy, vol. 27, no. 10, pp. 1758-1771, 2019.

[9] T. S. Elton, H. Selemon, S. M. Elton, and N. L. Parinandi, "Regulation of the MIR155 host gene in physiological and pathological processes," Gene, vol. 532, no. 1, pp. 1-12, 2013.

[10] H. Hu, X. Zhu, and X. Lin, "GuaLou GuiZhi decoction represses LPS-induced BV2 activation via miR-155 induced inflammatory signals," Pakistan Journal of Pharmaceutical Sciences, vol. 33, no. 1, pp. 403-408, 2020.

[11] H. Hu, X. Zhu, R. Lin, Z. Li, and L. Chen, "Suppressive effects of Gua Lou Gui Zhi decoction on MCAO-induced NO and PGE2 production are dependent on the MAPK and NF- $\kappa \mathrm{B}$ signaling pathways," Molecular Medicine Reports, vol. 14, no. 6, pp. 5141-5147, 2016.

[12] J. Matsuo, S. Haga, K. Hashimoto et al., "Activation of caspase-3 during Chlamydia trachomatis-induced apoptosis at a late stage," Canadian Journal of Microbiology, vol. 65, no. 2, pp. 135-143, 2019.

[13] A. Klegeris, "Regulation of neuroimmune processes by damage- and resolution-associated molecular patterns," Neural Regeneration Research, vol. 16, no. 3, pp. 423-429, 2021.

[14] I. A. Clark and B. Vissel, "Broader insights into understanding tumor necrosis factor and neurodegenerative disease pathogenesis infer new therapeutic approaches," Journal of Alzheimer's Disease, vol. 79, no. 3, pp. 931-948, 2021.

[15] N. Kerr, D. W. Dietrich, H. M. Bramlett, and A. P. Raval, "Sexually dimorphic microglia and ischemic stroke," CNS Neuroscience and Therapeutics, vol. 25, no. 12, pp. 1308-1317, 2019.

[16] S.-c. Zhao, L.-s. Ma, Z.-h. Chu, H. Xu, W.-q. Wu, and F. Liu, "Regulation of microglial activation in stroke," Acta Pharmacologica Sinica, vol. 38, no. 4, pp. 445-458, 2017.

[17] J. L. Marques-Rocha, M. Samblas, F. I. Milagro, J. Bressan, J. A. Martínez, and A. Marti, "Noncoding RNAs, cytokines, and inflammation-related diseases," The FASEB Journal, vol. 29, no. 9, pp. 3595-3611, 2015.

[18] S. Subramanian and C. J. Steer, "MicroRNAs as gatekeepers of apoptosis," Journal of Cellular Physiology, vol. 223, no. 2, pp. 289-298, 2010.

[19] G. Mahesh and R. Biswas, "MicroRNA-155: a master regulator of inflammation," Journal of Interferon and Cytokine Research, vol. 39, no. 6, pp. 321-330, 2019.

[20] X. H. Sun, M. F. Song, H. D. Song et al., "miR-155 mediates inflammatory injury of hippocampal neuronal cells via the activation of microglia," Molecular Medicine Reports, vol. 19, no. 4, pp. 2627-2635, 2019.

[21] S. S. Mortazavi-Jahromi, M. Aslani, S. Omidian et al., "Immunopharmacological effect of beta-d- mannuronic acid (M2000), as a new immunosuppressive drug, on gene expression of miR-155 and its target molecules (SOCS1, SHIP1) in a clinical trial on rheumatoid arthritis patients," Drug Development Research, vol. 81, no. 3, pp. 295-304, 2020.

[22] J. Yang, X. Zhu, H. Hu, and X. Lin, "The inhibitory effect of Gualou Guizhi decoction on post-ischemic neuroinflammation via miR-155 in MCAO rats," Annals of Palliative Medicine, vol. 10, no. 2, pp. 1370-1379, 2021.

[23] L. Nan, L. Yang, Y. Zheng et al., "Effects of gualou Guizhi decoction aqueous extract on axonal regeneration in organotypic cortical slice culture after oxygen-glucose deprivation," Evidence-Based Complementary and Alternative Medicine, vol. 2017, Article ID 5170538, 2017. 